\title{
Use of Transformations in Active BP Filter Designs
}

\author{
Neven Mijat and Dražen Jurišić \\ Faculty of Electrical Engineering and Computing, Unska 3, HR-10000 Zagreb, Croatia. \\ neven.mijat@ffer.hr; drazen.jurisic@ffer.hr
}

\begin{abstract}
In this paper transformations, which are very often used in design of active filters, are summarized, and used for revealing the relations between different classes of active filter sections. Among various transformations we distinguish: frequency transformations and network transformations. As frequency transformation examples we consider the well known "low-pass to band-pass" (LP-BP) and less known "lossy low-pass to bandpass"(LLP-BP) frequency transformation. As examples of network transformations, which are performed by network elements substitution we consider (i) "lossy" LP-BP network transformation: to obtain BP filter it substitutes each resistor of the LP prototype filter by a series $R C$ circuit, and each capacitor by a parallel $R C$ circuit; and (ii) complementary transformation: passive network in negative feedback loop is transformed into passive network in positive feedback loop of operational amplifier.
\end{abstract}

\section{Introduction}

The design of BP filters is usually performed using the well-known LP-BP frequency transformation applied to an LP prototype filter transfer function. Corresponding network LP-BP transformation can be applied only in the design of passive $L C$ filters. Since it is a reactance transformation resulting with the use of inductances, it can not be applied for the design of active filters. One possible solution is "lossy" LP-BP (LLP-BP) transformation which is presented in this paper [1].

Another network transformation we deal with, belongs to the group of complementary [2]-[4] transformations. It relates BP filter circuits based on positive feedback to those based on negative feedback. As a consequence their passive sensitivity properties are closely related, as well as their gain-sensitivity products (GSP). This transformation simplifies the filter realization procedures, as well as optimization of filter sensitivities.

\section{Lossy LP-BP Transformation}

The advantage of a passive- $L C \mathrm{BP}$ filter realization lies in the existence of reactance LP-BP transformation, which defines the BP filter structure and enables a straightforward realization procedure. An $L C \mathrm{BP}$ filter is obtained by replacing each inductor $L_{L P}$ of an LP prototype filter, by a series connection of $L_{a}$ and $C_{a}$ and each capacitor $C_{L P}$ by a parallel combination of $L_{b}$ and
$C_{b}$. Such an element transformation, gives a unique BP structure, following directly from LP-BP frequency transformation

$$
s_{L P}=\left(s^{2}+\omega_{0}^{2}\right) / B s .
$$

It transforms the complex frequency variable $s_{L P}$ into a rational function producing the transfer function of a BP filter with the center frequency $\omega_{0}$ and the bandwidth $B$.

The application of the LP-BP frequency transformation (1) to some $n^{\text {th }}$-order low-pass prototype filter gives a symmetrical $2 n^{\text {th }}$-order BP filter whose transfer function can be represented as a product of $n$ $2^{\text {nd }}$-order transfer functions

$$
T_{B P}(s)=\prod_{i=1}^{n} \frac{K_{i}\left(\omega_{i} / q_{i}\right) \cdot s}{s^{2}+\left(\omega_{i} / q_{i}\right) \cdot s+\omega_{i}^{2}} .
$$

LP-BP frequency transformation (1) is readily used in active BP filter designs, but application of the corresponding LP-BP reactance transformation makes no sense. One possible solution is a slight modification called "lossy" LP-BP transformation applied to the network realizing a translated LP prototype transfer function. The basic idea is the following. The original LP prototype transfer function is transformed by introducing the shifted complex frequency variable $p$

$$
p=s_{L P}+\delta,
$$

BP transfer function is created by applying the LLP-BP transformation to $p$, i.e.

$$
p=\frac{s^{2}+\omega_{0}^{2}}{B \cdot s}+\delta
$$

Note that the combination of (3) and (4) gives the same BP filter transfer function as the conventional LPBP transformation (1), applied to the original LP transfer function. However, a realization of a BP filter is enabled applying the corresponding impedance transformation to the modified LP prototype filter. The procedure can be briefly summarized through the following steps:

Given LP prototype transfer function $T(s)$

- Choose the shift constant $\delta$

- Find new transfer function $T_{1}(p)=T(s)=T_{1}(s+\delta)$

- Realize LP prototype having the transfer function $T_{1}(p)$

- Apply LLP-BP to $T_{1}(p)$ and obtain $T_{B P}(s)$

- Perform the LLP-BP impedance transformation to LP prototype filter.

The procedure is straightforward and it is described in more detail through the examples that follow.

\subsection{Second-Order BP filter}

Consider a $1^{\text {st }}$-order LP filter circuit in Fig. 1(a). 


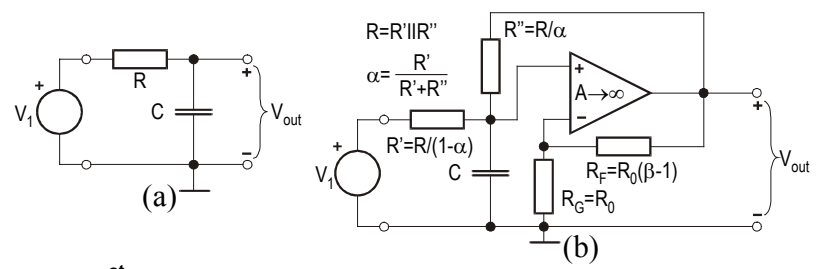

Fig. 1. $1^{\text {st }}$-order LP RC circuit. (a) passive (b) active

The voltage transfer function $T(s)$ for this circuit is

$$
T(s)=\frac{V_{o u t}}{V_{1}}=\frac{(R C)^{-1}}{s+(R C)^{-1}}=\frac{\sigma}{s+\sigma}=\frac{K}{\left(s-s_{p 1}\right)} .
$$

It has a negative real pole $s_{p 1}=-\sigma=-(R C)^{-1}$. Substituting

$$
s=p-\delta
$$

a new LP prototype function $T_{1}(p)$ is obtained

$$
\begin{gathered}
T_{1}(p)=\frac{\sigma}{s+\sigma}=\frac{\sigma}{p+\sigma-\delta}=\frac{\sigma}{p+\Gamma}=\frac{\sigma}{p-p_{p 1}} ; \\
p_{p 1}=-\Gamma \quad \Gamma=\sigma-\delta .
\end{gathered}
$$

The pole of $T_{1}(s)$ is shifted to the right for an amount $\delta$. It can be moved even in the right-half plane. In that case the $1^{\text {st }}$-order circuit with an operational amplifier, shown in Fig. 1(b), has to be used for realization of $T_{1}(s)$.
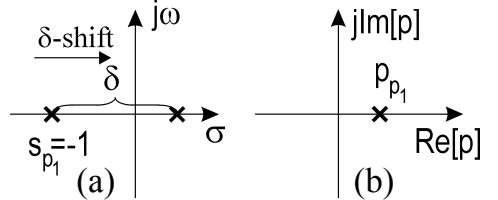

Fig. 2. $s$-variable translation. (a) Pole shift, (b) p-plane

The voltage transfer function $T_{1}(p)$ for this circuit is

$T_{1}(p)=\frac{V_{\text {out }}}{V_{1}}=\frac{(1-\alpha) \beta \sigma}{p+(1-\alpha \beta) \sigma}=\frac{(1-\alpha) \beta \sigma}{p+\sigma-\delta}=\frac{\beta \sigma-\delta}{p+\Gamma}$,

where the shift $\delta$ equals $\alpha \beta \sigma$. For the simplicity reasons we will use normalized elements of the LP prototype, i.e. $R=1, C=1$, giving $\sigma=1$ and $\delta=\alpha \beta$. The result is

$$
T_{1}(p)=\frac{V_{\text {out }}}{V_{1}}=\frac{(1-\alpha) \beta}{p+1-\alpha \beta}=\frac{\beta-\delta}{p+1-\delta} .
$$

At this point we introduce the impedance transformation which substitutes each resistor of the LP prototype filter by a series $R C$ circuit, and each capacitor by a parallel $R C$ circuit, as shown in Fig. 3 .

$$
\begin{aligned}
& R \rightarrow \frac{1 / R_{a}+s C_{a}}{1 / R_{a} \cdot s C_{a}}=\frac{1}{s C_{a}}+R_{a}, p C \rightarrow \frac{1}{R_{b}}+s C_{b} \\
& \mathrm{R} \rightarrow \mathrm{R}_{\mathrm{a}}
\end{aligned}
$$

Fig. 3. $R C$ impedance transformation.

Consequently the normalized variable $p$ of the LP transfer function is transformed into a rational function, i.e.

$$
p R C=\frac{p}{\sigma}=p=\frac{s^{2}+1 /\left(R_{a} C_{a} R_{b} C_{b}\right)}{s \cdot 1 /\left(R_{a} C_{b}\right)}+\frac{R_{a}}{R_{b}}+\frac{C_{b}}{C_{a}}
$$

Comparing (4) and (11) we obtain

$$
\omega_{0}^{2}=\frac{1}{R_{a} C_{a} R_{b} C_{b}}, B=\frac{1}{R_{a} C_{b}}, \delta=\frac{R_{a}}{R_{b}}+\frac{C_{b}}{C_{a}} .
$$

This procedure results by a $2^{\text {nd }}$-order BP filter shown in Fig. 4, which is a type A circuit described in [5].

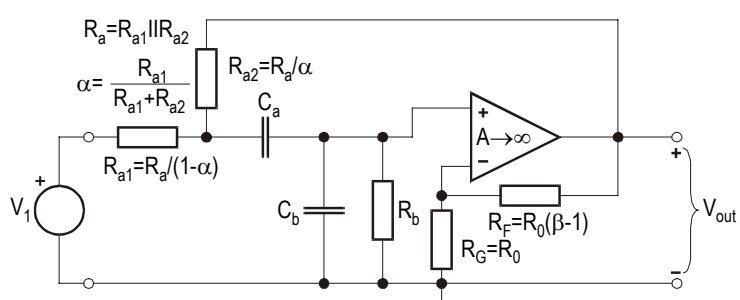

Fig. 4. Second-order active-RC BP filter circuit.

The voltage transfer function $T_{B P}(s)$ of this circuit is

$$
T_{B P}(s)=\frac{V_{\text {out }}}{V_{1}}=\frac{(1-\alpha) \beta}{\frac{s^{2}+\omega_{0}^{2}}{B s}+\delta-\alpha \beta+1}=\frac{(1-\alpha) \beta B s}{s^{2}+B s+\omega_{0}^{2}}
$$

The value of $\delta$ is not arbitrary. It is limited by the resistance and capacitance ratios. From (12) we have

$$
\frac{R_{a}}{R_{b}}=\frac{\delta}{2} \mp \sqrt{\frac{\delta^{2}}{4}-\frac{\omega_{0}^{2}}{B^{2}}} \frac{C_{b}}{C_{a}}=\frac{\delta}{2} \pm \sqrt{\frac{\delta^{2}}{4}-\frac{\omega_{0}^{2}}{B^{2}}} .
$$

The expression under the square roots is positive if

$$
\delta \geq 2 \omega_{0} / B=\delta_{\min }=2 q_{p} .
$$

The constant $\delta$ is, therefore, limited by the ratio of the central frequency and the bandwidth of the BP filter. There are many degrees of freedom in realization of (13) by choosing parameters $\alpha, \beta$ and $\delta$. The main criterion is to minimize the transfer function sensitivities. As shown in [1], this is the case when (15) is satisfied, i.e.

$$
C_{b} / C_{a}=R_{a} / R_{b}=\delta_{\text {min }} / 2 \text {. }
$$

Design Example: As an illustration consider a $2^{\text {nd }}$-order BP filter, with the center frequency $f_{0}=1 \mathrm{kHz}$, bandwidth $B=500 \mathrm{~Hz}$, and the pass-band gain $K=1$. The design is performed by the following design procedure:

i) For a given $2^{\text {nd }}$-order BP filter pole $Q$, choose $\delta$. Using (15) we calculate

$$
\delta_{\text {min }}=2 \omega_{0} / B=2 q_{p}=4 .
$$

Let us denote the capacitance ratio as $\rho,=C_{b} / C_{a}$, and the resistance ratio as $r=R_{a} / R_{b}$. In order to analyze their influence on sensitivities to component tolerances, and to find an optimal $\delta$, three realizations corresponding to two values of $\delta$ are analyzed. First we choose $\delta=\delta_{\min }=4$, and from (16) we have $\rho=r=\delta_{\min } / 2=2$ (circuit 1). Next we choose $\delta=6$ and using (14) obtain $\rho=5.236$ and $r=0.764$ for plus sign of the square root (circuit 2), and $\rho=0.764$ and $r=5.236$ (circuit 3 ) for minus sign. Let us proceed with calculation of circuit no 2 .

ii) Find the new LP prototype with shifted poles by $\delta$ : Using (6), a new function $T_{1}(p)$ with $\Gamma=-5$ is obtained. To realize negative $\Gamma$ we have to calculate gain $\beta$. Choosing $\alpha=0.5$, from (9) it follows $\beta=\delta / \alpha=12$.

iii) Realize BP filter circuit components.

We choose $C_{b}=5 \mathrm{nF}$, and using (12) $C_{a}=C_{b} / \rho=955 \mathrm{pF}$, $R_{a}=1 /\left(B \cdot C_{b}\right)=63.7 \mathrm{k} \Omega$ and $R_{b}=B /\left(C_{a} \cdot \omega_{0}^{2}\right)=83.3 \mathrm{k} \Omega$. The remaining elements are: $R_{a 1}=R_{a} /(1-\alpha)=127 \mathrm{k} \Omega ; R_{a 2}=$ $R_{a} / \alpha=127 \mathrm{k} \Omega$. Let $R_{G}=1 \mathrm{k} \Omega$, then $R_{F}=R_{G}(\beta-1)=11 \mathrm{k} \Omega$. The element values for this case and for the other two are given in Table 1. Fig. 5(a) shows the transfer function magnitude $\alpha(\omega)=20 \log |T(j \omega)|[\mathrm{dB}]$. 


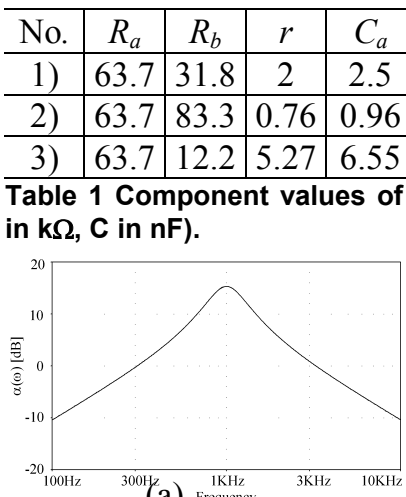

(a) ${ }_{\text {Frequency }}^{1 \mathrm{KHz}}$

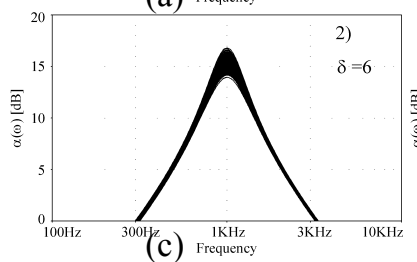

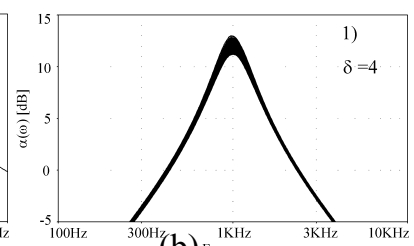

(b) ${ }_{\text {Frequency }}^{\mathrm{K} H z}$

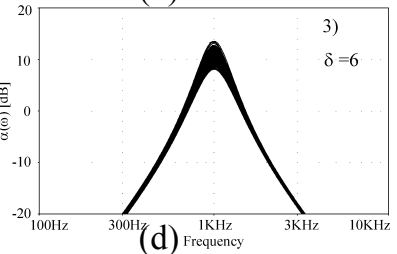

Fig. 5 (a) Magnitude, and (b)-(d) results of Monte Carlo analysis of the filters in Table 1.

Monte Carlo simulation results are presented in Fig. 5(b)-(d). The relative element changes are assumed as uncorrelated random variables, with a zero-mean Gaussian distribution and 1\% standard deviation.

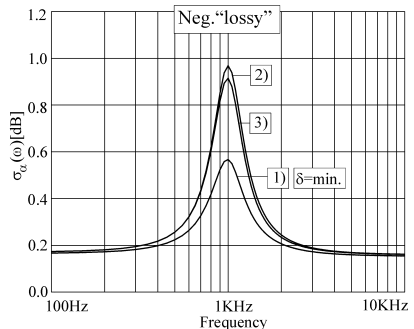

Fig. 6 Sensitivities of the BP filters in Table 1.

The standard deviation (related to the Shoeffler sensitivities) of the gain variation with respect to the elements variations $\Delta \alpha=8.68588 \Delta\left|T_{B P}(\omega)\right| /\left|T_{B P}(\omega)\right|$, is calculated for the filter examples in Table 1 and shown in Fig. 6. We see that the best results are obtained if $\delta=\delta_{\min }$, i.e. for the circuit no. 1, while the two remaining circuits have similar but worse sensitivities.

\subsection{Fourth-Order BP filter}

Consider the $2^{\text {nd }}$-order LP filter shown in Fig. 7.

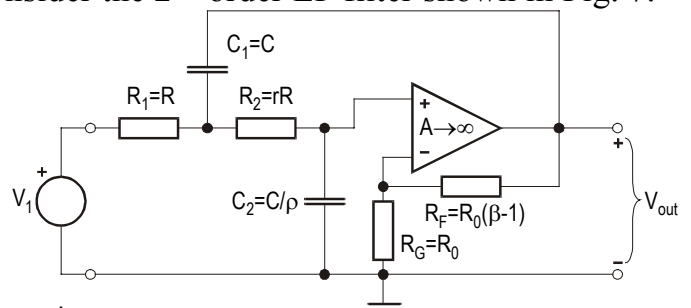

Fig. 7. $2^{\text {nd }}$-order low-pass filter.

The BP filter shown in Fig. 8 is obtained by the element substitution $(10)$ to that circuit. Since the $2^{\text {nd }}-$ order LP filter prototype for the design of $4^{\text {th }}$-order BP filter, has a complex pole pair we do not need to shift any real poles, and modify it as we did in the $1^{\text {st }}$-order LP prototype case. As shown in [1] minimal $\delta$ provides the low sensitive circuit to the component tolerances. Further sensitivity reduction can be achieved applying the "impedance tapering" to the LP prototype [6].

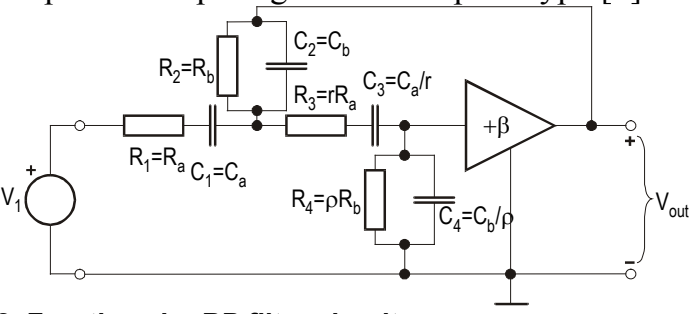

Fig. 8. Fourth-order BP filter circuit

\subsection{Higher-Order BP filter}

High-order BP filters can be realized using the same procedure. There is, however, a difference between the filters that use odd-order and even-order LP prototype.

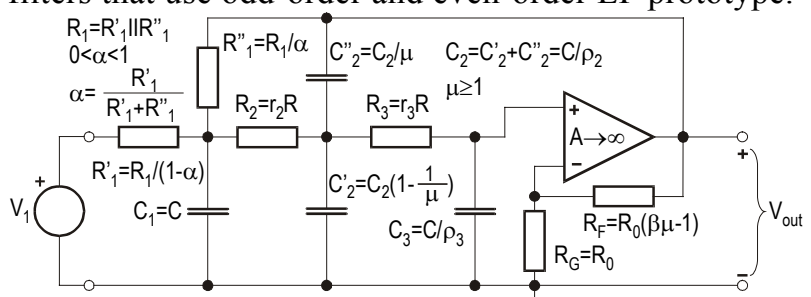

Fig. 9. Modified $3^{\text {rd }}$-order LP filter prototype

To construct a $6^{\text {th }}$-order BP filter, a $3^{\text {rd }}$-order LP prototype filter shown in Fig. 9 is used.

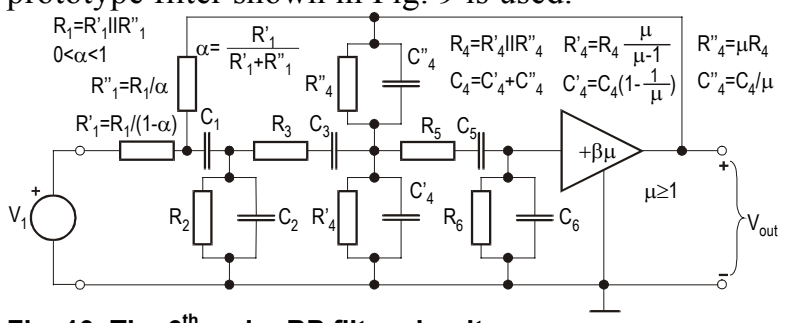

Fig. 10. The $6^{\text {th }}$-order BP filter circuit

It is a slightly modified standard single-amplifier $3^{\text {rd }}$ order LP filter configuration. Feedback resistor $R_{1}$ '" is added to enable a shift of a real pole together with complex poles. Resulting BP filter is shown in Fig. 10.

A $4^{\text {th }}$-order LP prototype for the realization of an $8^{\text {th }}$ order BP filter is shown in Fig. 11. Its transfer function has only complex-conjugate poles and their $\delta$-shift needs no additional feedback connections.

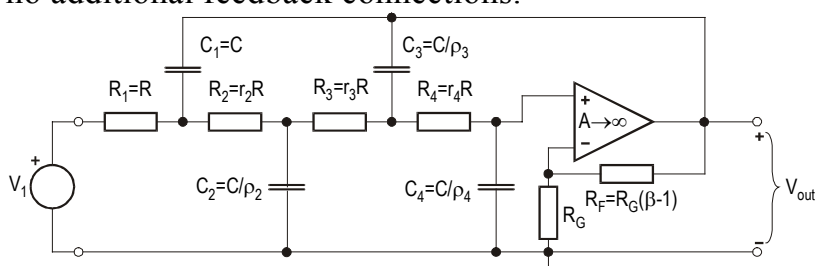

Fig. 11. The $4^{\text {th }}$-order LP prototype filter.

The element substitution (10) applied to this circuit, gives the BP filter shown in Fig. 12.

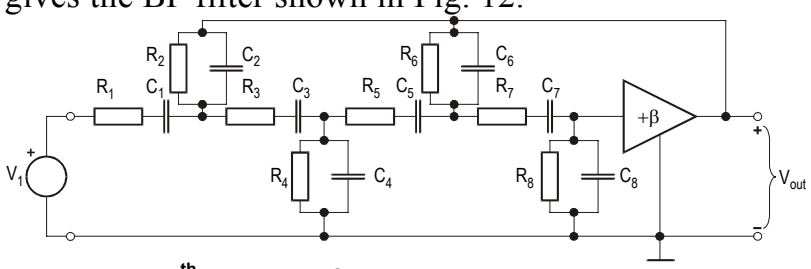

Fig. 12. The $8^{\text {th }}$-order BP filter circuit. 


\section{Complementary transformation}

Evaluation of active- $R C$ filter quality involves various parameters such as: simple realizability, repeatability, a possibility of straightforward procedure of parameter calculation, small number of components, low power consumption, low noise, and the most often, low filter sensitivity to passive and active component tolerances.

Active- $R C$ single-amplifier filters with $R C$ network in the feedback loop satisfy most of these performances. However, calculation of filter elements and optimization of sensitivities can be very tedious and time consuming.

Some filter configurations can be obtained from another ones with known performance using certain network transformations. Also, if the original filter is optimized, the transformation can give an optimized circuit as well, making the realization procedure simple. We pay an attention to a transformation belonging to a group of complementary transformations [3], [4]. As an example a $2^{\text {nd }}$-order active BP filter section is used.

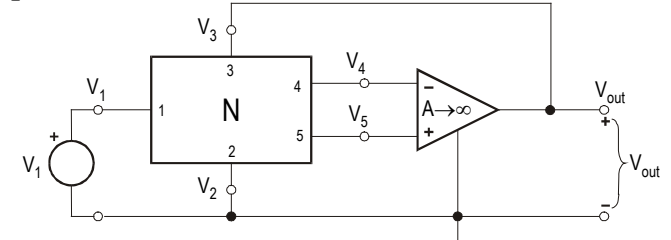

Fig. 13. Single operational amplifier active filter

Second-order active filters are often realized as a single amplifier form shown in Fig. 13, with an $R C$ network in the negative feedback loop. Using the equivalent circuit shown in Fig. 14 we can write the equations for the voltages at the nodes 4 and 5

$$
\begin{aligned}
& V_{4}=t_{41} V_{1}+t_{42} V_{2}+t_{43} V_{3} \\
& V_{5}=t_{51} V_{1}+t_{52} V_{2}+t_{53} V_{3}
\end{aligned}
$$

where

$$
t_{i j}=\left.\frac{V_{i}}{V_{j}}\right|_{V_{k}=0} \quad ; \quad i=4,5 \quad, \quad j=1,2,3 \quad, \quad k \neq j
$$

are the voltage transfer functions corresponding to $j^{\text {th }}$ input and $i^{\text {th }}$ output node of network N. It is known that [4]

$$
\sum_{i=1}^{3} t_{i j}=1 ; \quad i=4,5 \text {. }
$$

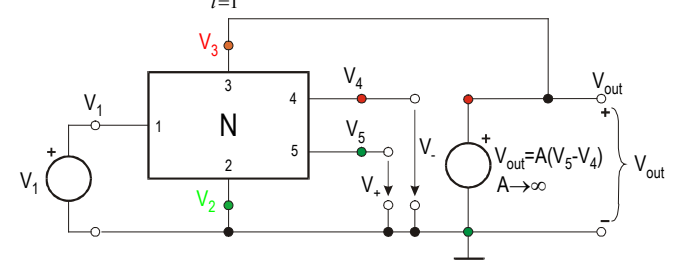

Fig. 14. Active filter section equivalent circuit.

Different configurations and transfer functions of the complete section can be obtained by connecting the input nodes 1,2 , or 3 to the section input or amplifier's output or to the ground. We shall consider two cases.

Case I: First we consider a circuit presented in Fig. 13, where the node 1 is the input, 2 is ground node and node 3 is connected to the operational amplifier output, i.e.

$$
V_{1}=V_{\text {in }} ; V_{2}=0 ; V_{3}=V_{\text {out }}
$$

$V_{4}$ and $V_{5}$ are the voltages at the virtual short circuit, i.e.

$$
V_{4} \cong V_{5} \text {. }
$$

Using (18), (21) and (22) the overall transfer function is

$$
T_{1}(s)=\frac{V_{\text {out }}}{V_{\text {in }}}=\frac{V_{3}}{V_{1}}=-\frac{t_{51}-t_{41}}{t_{53}-t_{43}}
$$

Case II: If we interchange the output and ground terminal of the circuit from Fig. 14, we obtain a circuit in Fig. 15, with the node 2 of the network $N$ connected to the operational amplifier output and grounded node 3 .

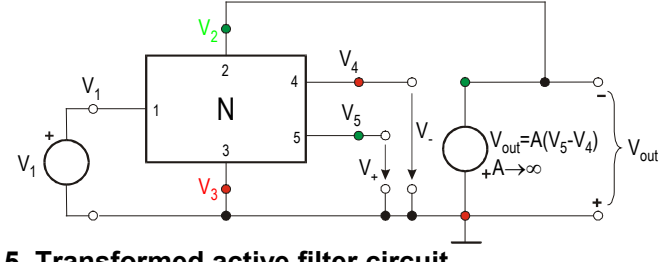

Fig. 15. Transformed active filter circuit.

Note that the voltage source must also be inverted in the process of exchanging terminals. This cannot be done in practice, but the equivalent result, i.e. reversing the output voltage polarity, can be achieved by inverting the input terminals of the operational amplifier. Therefore, the practical circuit has the form shown in Fig. 16. For this section we can write

$$
V_{1}=V_{\text {in }} \quad V_{2}=V_{\text {out }} \quad V_{3}=0
$$

Combining (18), (24) and (22) gives

$$
T_{2}(s)=\frac{V_{\text {out }}}{V_{\text {in }}}=\frac{V_{2}}{V_{1}}=-\frac{t_{51}-t_{41}}{t_{52}-t_{42}}
$$

Fig. 16. Complementary active filter section.

Comparing (23) and (25) and applying (20) we get

$$
\frac{1}{T_{1}}+\frac{1}{T_{2}}=1
$$

i.e. transfer functions reciprocals are complementary. They have different denominators and equal numerators. Therefore, if the first circuit is a BP filter, another one is a BP filter as well.

The representative examples are two well-known BP $2^{\text {nd }}$-order circuits. The circuit in Fig. 17 is the SingleAmplifier-Biquad (SAB), with an $R C$ network in the negative feedback loop of the operational amplifier and corresponds to the topology presented in Case I above.

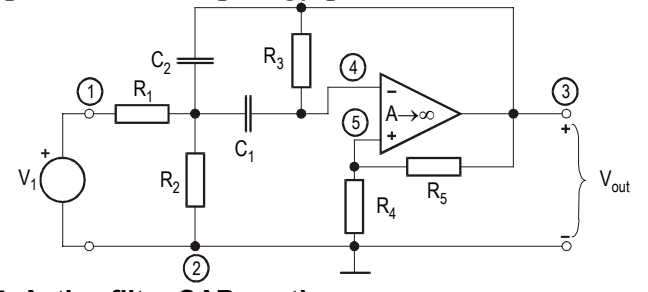

Fig. 17. Active filter $S A B$ section

Complementary to the SAB section is Sallen and Key (SAK) section shown in Fig. 18. 


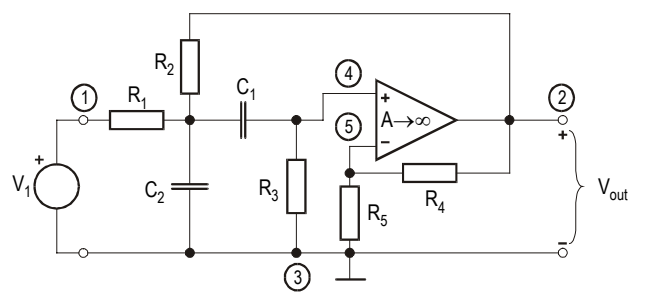

Fig. 18. Complementary SAK active filter section

SAK section uses positive feedback of the operational amplifier and corresponds to the Case II above. Generally both circuits have the transfer function

$$
T(s)=\frac{V_{\text {out }}}{V_{1}}=\frac{N(s)}{D(s)}=K \frac{a_{1} s}{s+a_{1} s+a_{0}}=K \frac{\frac{\omega_{p}}{q_{p}} s}{s+\frac{\omega_{p}}{q_{p}} s+\omega_{p}^{2}}
$$

Assuming that passive $R C$ networks in the amplifier's positive (SAB) and negative (SAK) feedback loops are equal, we will use the following transfer function of the passive $R C$ network

$$
\hat{T}(s)=\frac{n(s)}{d(s)}=\hat{k} \frac{\frac{\omega_{p}}{\hat{q}} s}{s+\frac{\omega_{p}}{\hat{q}} s+\omega_{p}^{2}},
$$

where

$$
\begin{gathered}
\hat{k}=\frac{R_{3} C_{1}}{R_{p}\left(C_{1}+C_{2}\right)+R_{3} C_{1}} ; \quad \hat{q}=\frac{\sqrt{R_{p} C_{1} R_{3} C_{2}}}{R_{p}\left(C_{1}+C_{2}\right)+R_{3} C_{1}} ; \\
\omega_{p}=\frac{1}{\sqrt{R_{p} C_{1} R_{3} C_{2}}} ; \quad R_{p}=R_{1} \| R_{2}=\frac{R_{1} R_{2}}{R_{1}+R_{2}} .
\end{gathered}
$$

The transfer function for the SAB circuit has the form

$$
T_{1}(s)=\frac{N(s)}{D(s)}=-\frac{\alpha_{1} \beta_{1} \hat{T}(s)}{1-\beta_{1} \hat{T}(s)}
$$

and for the SAK circuit

$$
T_{2}(s)=\frac{N(s)}{D(s)}=\frac{\alpha_{2} \beta_{2} \hat{T}(s)}{1-\beta_{2}\left(1-\alpha_{2}\right) \hat{T}(s)}
$$

They must be identical, i.e. $T_{1}(s)=T_{2}(s)=T(s)$, and comparing their numerators and denominators we obtain

$$
\alpha_{1}=\alpha_{2} /\left(1-\alpha_{2}\right) ; \quad \beta_{1}=\beta_{2}\left(1-\alpha_{2}\right) .
$$

Finally, the coefficients of the transfer function (27) are given by

$$
\begin{gathered}
K_{i}=(-1)^{i} \alpha_{i} \beta_{i} \hat{k} q_{p i} / \hat{q} ; i=1,2 . \\
\omega_{p i}=\omega_{p} ; i=1,2 . \\
q_{p i}=\hat{q} /\left(1-b_{i} \hat{k}\right) ; i=1,2 .
\end{gathered}
$$

where $b_{1}=\beta_{1}$, and $b_{2}=\beta_{2}\left(1-\alpha_{2}\right)$.

Design Example: As an illustration consider a $2^{\text {nd }}$-order BP filter with $f_{0}=1 \mathrm{kHz}, B=500 \mathrm{~Hz}$ and $K=1$.

For the SAB design we choose $\rho=C_{2} / C_{1}=1, r=R_{3} / R_{p}=1$, $C_{2}=5 \mathrm{nF}$. Other components following from (28b) are given in line 1 of Table 2 .

\begin{tabular}{c|c|c|c|c|c|c|c|c|c|c}
\hline No. & $R_{1}$ & $R_{2}$ & $\alpha_{1}$ & $R_{p}$ & $R_{3}$ & $r$ & $C_{1}$ & $C_{2}$ & $\rho$ & $\beta_{1}$ \\
\hline 1$)$ & 159 & 39.8 & 0.2 & 31.8 & 31.8 & 1 & 5 & 5 & 1 & 2.5 \\
\hline 2$)$ & 114 & 44.6 & .286 & 31.8 & 127 & 4 & 1.25 & 5 & 4 & 1.75 \\
\hline
\end{tabular}

Table 2. SAB filter component values ( $R$ in $\mathrm{k} \Omega, \mathrm{C}$ in $\mathrm{nF}$ ).

From (32c) $\beta_{1}=2.5$, and with $R_{5}=1 \mathrm{k} \Omega$ we calculate $R_{4}=\left(\beta_{1}-1\right) \cdot R_{5}=1.5 \mathrm{k} \Omega$. From (32a) $\alpha_{1}=0.2$. Finally we calculate $\quad R_{1}=R_{p} / \alpha_{1}=159.15 \mathrm{k} \Omega, \quad$ and $\quad R_{2}=R_{p} /(1-$ $\left.\alpha_{1}\right)=39.79 \mathrm{k} \Omega$. Filter no. 2 in Table 2 is the "ideal impedance tapering" realization, i.e. with $\rho=r=4$.

The design of the SAK section is now very simple. Using the elements of the SAB section from Table 2, we only need to calculate the new values of constants $\alpha$ and $\beta$. Thus for circuit no. 1 using (31) we obtain

$$
\alpha_{2}=\alpha_{1} / \alpha_{1}+1=0.1667 ; \quad \beta_{2}=\beta_{1}\left(\alpha_{1}+1\right)=3 \text {. }
$$

The resistors $R_{1}, R_{2}$, and $R_{4}$ are: $R_{1}=R_{p} / \alpha_{2}=191 \mathrm{k} \Omega$, $R_{2}=R_{p} /\left(1-\alpha_{2}\right)=38.2 \mathrm{k} \Omega, R_{4}=\left(\beta_{2}-1\right) \cdot R_{5}=2 \mathrm{k} \Omega$. For SAK no. 2 we have $\alpha_{2}=0.222, \quad \beta_{2}=2.25, \quad R_{1}=143.24 \mathrm{k} \Omega$, $R_{2}=40.93 \mathrm{k} \Omega$, and $R_{4}=1.25 \mathrm{k} \Omega$.

Active sensitivities can be reduced minimizing the GSP in the filter design. Passive sensitivities are reduced using "impedance tapering" [7]. It can be shown that optimization of SAB circuit gives the optimum for SAK circuit as well [8]. The results of using Monte Carlo simulations for the SAB section, are shown in Fig. 19. Better results are obtained for the impedance-tapered circuit, i.e. for the circuit no. 2.

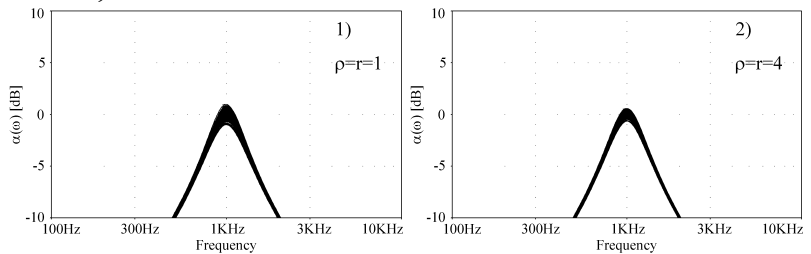

Fig. 19 Monte Carlo runs of SAB BP filters

MC runs for the SAK section are presented in Fig. 20. Better sensitivity is obtained for the circuit no. 2, again.

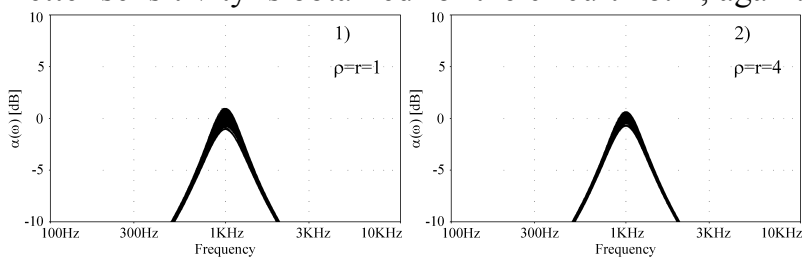

Fig. 20 Monte Carlo runs of SAK BP filters

Shoeffler sensitivities of both SAB and SAK circuits are presented in Fig. 21. We can conclude that two complementary circuits in Fig. 17 and Fig. 18 have very similar characteristics concerning sensitivity to passive filter components. SAB circuit shows somewhat lower sensitivity.

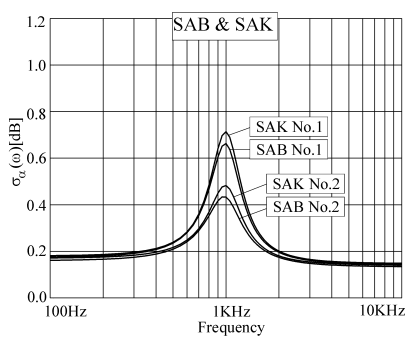

Fig. 21 Shoeffler sensitivities of SAB and SAK filters

\section{Combining L-LP-BP and Complementary transformations}

Consider the $1^{\text {st }}$-order circuit with an operational amplifier shown in Fig. 1(b). If we apply complementary transformation to this circuit we obtain the circuit in Fig. 
22 , with an $R C$ network in the negative feedback loop. The voltage transfer function $T_{2}(p)$ for this circuit is

$$
T_{2}(p)=\frac{V_{\text {out }}}{V_{1}}=-\frac{(1-\alpha) \beta \sigma}{p+(1-\beta) \sigma}=\frac{(1-\alpha) \delta}{p+\sigma-\delta}=\frac{(1-\alpha) \Gamma}{p+\Gamma} .
$$

Fig. 22. Complementary $1^{\text {st }}$-order shifted pole circuit

We choose again $\sigma=1$ for simplicity. The transfer function $T_{2}(p)$ as defined by (34) has the same form as $T_{1}(p)$ in (8). To realize $T_{1}(p)=T_{2}(p)$ their numerators and denominators must be identical, i.e.

$$
\beta_{2}=\alpha_{1} \beta_{1} ; \alpha_{2}=2-1 / \alpha_{1} \text {. }
$$

To provide the same pole shift for value of $\delta$, as in the positive feedback case, we need to calculate a gain $\beta_{2}$.

Application of L-LP-BP transformation to that circuit gives a new BP filter circuit shown in Fig. 23. It is complementary to the one in Fig. 4 and we expect that it has sensitivity properties very similar to the original circuit. Consequently, optimizing GSP and minimizing sensitivity to passive components by using minimum $\delta$ in filter design can be inherited from the original circuit.

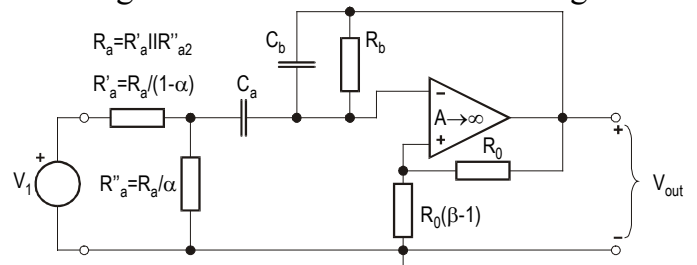

Fig. 23. Complementary $2^{\text {nd }}$-order active-RC BP circuit.

For the circuits in Table 1 we calculated new values for gain $\beta$. Thus for no. 1 using (35) we have $\beta_{2}=4$, and for circuits no. 2,3 we have $\beta_{2}=6$. We used element values from Table 1 and new gain $\beta$ to built circuits as in Fig. 23. Monte Carlo runs and Shoeffler sensitivities are presented in Fig. 24 and Fig. 25, respectively. We can conclude that min. sensitivity is obtained for the circuit no.1 (minimum $\delta$ ), again. Two complementary circuits in Fig. 4 and Fig. 23 have very similar sensitivity properties to passive components.
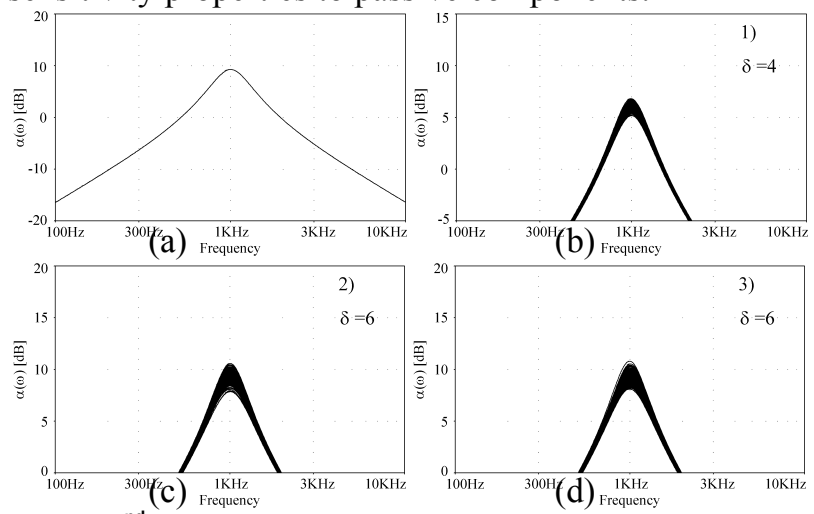

Fig. $242^{\text {nd }}$-order BP filter as in Fig. 23: (a) Magnitude. (b)-(d) Monte Carlo runs.

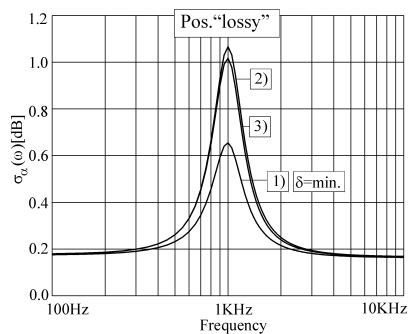

Fig. 25 Sensitivities of $2^{\text {nd }}$-order BP filters from Fig. 23.

Comparing all realized $2^{\text {nd }}$-order BP filters it can be concluded that both $\mathrm{SAB}$ and SAK topologies have better sensitivities than topologies obtained using L- LP$\mathrm{BP}$ transformation. In all cases filters with negative feedback always have lower sensitivities.

\section{Conclusions}

Lossy LP-BP transformation enables BP filter realizations using direct component transformation and straightforward design procedure. Complementary transformation relates the designs, which use a passive filter sub-network in positive and negative feedback loop of OA. Component values calculated for one topology can be used for calculation of the complementary topology circuit. Their Gain-SensitivityProducts (GSP) and passive sensitivity performances are also closely related.

\section{References}

[1] D. Jurišić, N. Mijat G. S. Moschytz, "Design of $4^{\text {th }}$ Order Band-Pass Active-RC Filters Using "Lossy" LP-BP Transformation," Proc. ECCTD 2001, Espoo, Finland, Vol. 1, Aug. 28 - 31, 2001, pp. 117-120.

[2] G. S. Moschytz, P. Horn, "Optimizing Two Commonly Used Active-Filter Building Blocks Using the Complementary Transformation," Electronic Circ. and Syst., vol. 1, no. 4, pp. 125-132, July 1977.

[3] N. Fliege, "Complementary Transformation of Feedback Systems", IEEE Trans. on Circuit Theory, CT-20, pp. 137-139, 1973.

[4] D. Hilberman, "Input and Ground as Complements in Active Filters", IEEE Trans. on Circuit Theory, CT20, pp. 540-547, 1973.

[5] G. S. Moschytz, Linear Integrated Networks: Design. New York: Van Nostrad Reinhold Co., 1975.

[6] D. Jurišić, G.S. Moschytz, N. Mijat, "Low-Sensitivity, Low-Power $4^{\text {th }}$-Order Band-Pass Active-RC Allpole Filter Using Impedance Tapering,” Proc. ICECS '01, Msida, Malta, Vol. 2, Sept. 2-5, 2001, pp. 815-818.

[7] D. Jurišić, G. S. Moschytz, N. Mijat "Low-Sensitivity SAB Band-Pass Active-RC Filter Using Impedance Tapering," Proc. of the IEEE Int. Symp. on Circ. and Syst., ISCAS 2001, Sydney, Australia, Vol. 1, pp. 160163, May 6-9, 2001.

[8] N. Mijat, V. Čosić, "Complementary Transformations and Sensitivity of Active Filter Sections", InfTelecom-Automation ITA No. 3-4, vol. 8, pp. 309-325, July-Dec., 1989. 